This item was submitted to Loughborough's Research Repository by the author.

Items in Figshare are protected by copyright, with all rights reserved, unless otherwise indicated.

\title{
Heterogeneous domain decomposition methods for fluid-structure interaction problems
}

PLEASE CITE THE PUBLISHED VERSION

http://dx.doi.org/10.1007/978-3-540-34469-8_4

\section{PUBLISHER}

Springer

VERSION

NA (Not Applicable or Unknown)

\section{PUBLISHER STATEMENT}

This work is made available according to the conditions of the Creative Commons Attribution-NonCommercialNoDerivatives 4.0 International (CC BY-NC-ND 4.0) licence. Full details of this licence are available at: https://creativecommons.org/licenses/by-nc-nd/4.0/

\section{LICENCE}

CC BY-NC-ND 4.0

\section{REPOSITORY RECORD}

Deparis, Simone, Marco Discacciati, Gilles Fourestey, and Alfio Quarteroni. 2019. "Heterogeneous Domain Decomposition Methods for Fluid-structure Interaction Problems”. figshare. https://hdl.handle.net/2134/18754. 


\title{
Heterogeneous Domain Decomposition Methods for Fluid-Structure Interaction Problems
}

\author{
Simone Deparis ${ }^{1}$, Marco Discacciati ${ }^{2}$, Gilles Fourestey ${ }^{2}$, and Alfio \\ Quarteroni $^{2,3}$ \\ 1 Mechanical Engineering Department, Massachusetts Institute of Technology, 77 \\ Mass Ave, Cambridge MA 02139, USA. simone.deparis@epfl.ch \\ 2 IACS - Chair of Modeling and Scientific Computing, EPFL, CH-1015 Lausanne, \\ Switzerland. marco.discacciati@oeaw.ac.at, gilles.fourestey@epfl.ch \\ 3 MOX, Politecnico di Milano, P.zza Leonardo da Vinci 32, 20133 Milano, Italy. \\ alfio.quarteroni@epfl.ch
}

Summary. In this note, we propose Steklov-Poincaré iterative algorithms (mutuated from the analogy with heterogeneous domain decomposition) to solve fluidstructure interaction problems. Although our framework is very general, the driving application is concerned with the interaction of blood flow and vessel walls in large arteries.

\section{Introduction}

Mathematical modeling of real-life problems may lead to different kind of boundary value problems in different subregions of the original computational domain. The reason may be twofold.

Often, in order to reduce the computational cost of the simulation, a very detailed model can be used only in a region of specific interest while resorting to a simplified version of the same model sufficiently far away from where the most relevant physical phenomena occur. This is, e.g., the strategy adopted when one considers the coupling of advection-diffusion equations with advection equations, after neglecting the diffusive effects in a certain subregion (see, e.g., [11]), or when the full Navier-Stokes equations are coupled with Oseen, Stokes or even velocity potential models, the latter being adopted where the nonlinear convective effects are negligible (see, e.g., $[7,8]$ ).

In a second circumstance, one may be obliged to consider truly different models to account for the presence of distinct physical problems within the same global domain. This case is usually indicated as multi-physics or multifield problem. 
Typical examples are given by filtration processes such as in biomechanics or in environmental applications where a fluid (e.g. blood or water) can filtrate through a porous medium (e.g. the arterial wall or the soil), so that the NavierStokes equations must be coupled with Darcy's (or more complicated models, e.g., Forchheimer or Brinkmann equations) to describe the underlying physics (see, e.g., $[5,10,16,23]$ ).

All these problems may be cast into the same common framework of heterogeneous domain decomposition method, which extends the classical domain decomposition theory whenever two (or more) kinds of boundary value problems, say $L_{i} u_{i}=f_{i}$, hold in subregions $\Omega_{i}$ of the computational domain $\Omega$.

A major role is played by the compatibility conditions that the unknowns $u_{i}$ must satisfy across the interface which separates the subdomains. In fact, the setting of proper coupling conditions is a crucial issue to model as closely as possible the real physical phenomena. For example, when coupling the Navier-Stokes and the Oseen equations, the compatibility conditions require the continuity of the velocities and of the normal stresses across the interface. However, it is worth mentioning that they might be much less intuitive and easy to handle than in the case just mentioned (see, e.g., [5, 25]).

In this paper, we will apply the heterogeneous domain decomposition paradigm to a fluid-structure interaction problem arising in hemodynamics for modeling blood flows in large arteries. To preserve stability one should solve exactly the fluid-structure coupling, e.g. by Newton methods $[9,13]$ or fixed-point algorithms [2]. A Newton method with exact Jacobian has been investigated both mathematically and numerically in [9]. Segregated solvers yielding a single fluid-structure interaction in each time step do not preserve stability and may produce blow-up when the density of the structure stays below a critical threshold. On the other hand, to relax the computational complexity of fixed-point or Newton methods several inexact solution strategies can be adopted.

The Jacobian matrix can be simplified by dropping the cross block expressing the sensitivity of the fluid state to solid motion, or by replacing it by a simpler term that models added-mass effect (see [1, 12]). Alternative inexact solvers exploit the analogy of the fluid structure coupled problem with heterogeneous domain decomposition problems.

This approach was first presented in $[24,19]$ for a Stokes-linearized shell coupling and later studied also in [18], where the whole problem was first reformulated as an interface equation. In this paper we further pursue this approach. Iterative substructuring methods, typical of the domain decomposition approach, are used to solve the interface problem, exploiting the classical Dirichlet-Neumann, the Neumann-Neumann, or more sophisticated scaling (preconditioning) techniques.

After describing a precise setting of the problem (Sect. 2), we shall define the associated interface equation (Sect. 3) and illustrate possible iterative methods to solve it (Sect. 4). Finally, some numerical results will be presented (Sect. 5). 


\section{Problem setting}

To describe the evolution of the fluid and the structure domains in time, we adopt the ALE (Arbitrary Lagrangian Eulerian) formulation for the fluid (see $[6,14])$ and a purely Lagrangian framework for the structure. We denote by $\Omega(t)$ the moving domain composed of the deformable structure $\Omega^{\mathrm{s}}(t)$ and the fluid subdomain $\Omega^{\mathrm{f}}(t)$. If we denote by $\boldsymbol{d}^{\mathrm{s}}\left(x_{0}, t\right)$ the displacement of the solid
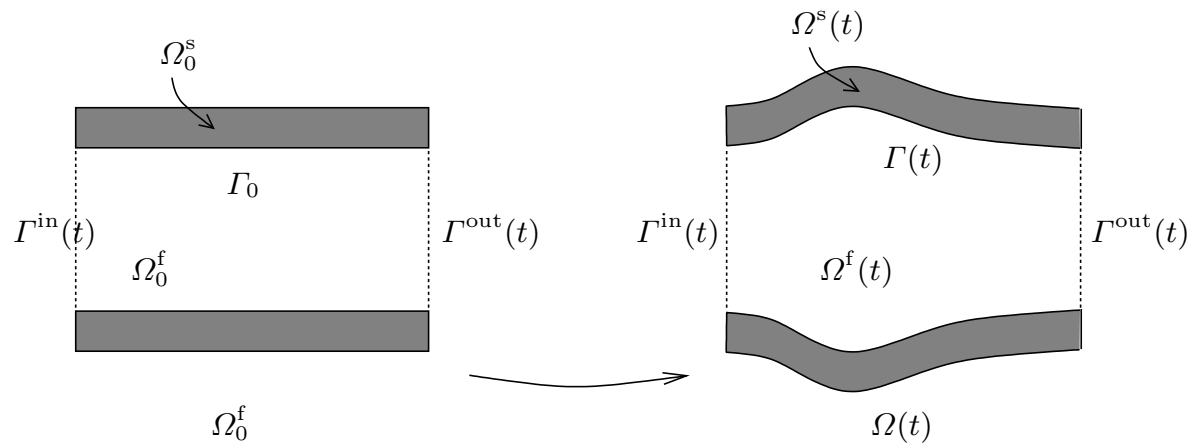

Fig. 1. ALE mapping

at a time $t$, we can define the following mapping: $\forall t, \Omega_{0}^{\mathrm{s}} \rightarrow \Omega^{\mathrm{s}}(t)$,

$$
x_{0} \rightarrow \boldsymbol{x}_{t}^{\mathrm{s}}\left(x_{0}\right)=x_{0}+\boldsymbol{d}^{\mathrm{s}}\left(x_{0}, t\right), \quad x_{0} \in \Omega_{0}^{\mathrm{s}} .
$$

Likewise, for the fluid domain: $\forall t, \Omega_{0}^{\mathrm{f}} \rightarrow \Omega^{\mathrm{f}}(t)$,

$$
x_{0} \rightarrow \boldsymbol{x}_{t}^{\mathrm{f}}\left(x_{0}\right)=x_{0}+\boldsymbol{d}^{\mathrm{f}}\left(x_{0}, t\right), \quad x_{0} \in \Omega_{0}^{\mathrm{f}} .
$$

The fluid domain displacement $\boldsymbol{d}^{\mathrm{f}}$ can be defined as a suitable extension of the solid interface displacement $\boldsymbol{d}_{\mid \Gamma_{0}}^{\mathrm{s}}: \boldsymbol{d}^{\mathrm{f}}=\operatorname{Ext}\left(\boldsymbol{d}_{\mid \Gamma_{0}}^{\mathrm{s}}\right)$ (see, e.g., [20]).

We assume the fluid to be Newtonian, viscous and incompressible, so that its behavior is described by the following fluid state problem: given the boundary data $\boldsymbol{u}_{\text {in }}, \boldsymbol{g}_{\mathrm{f}}$, and the forcing term $\boldsymbol{f}_{\mathrm{f}}$, and denoting $\boldsymbol{w}^{\mathrm{f}}=\partial_{t} \boldsymbol{d}^{\mathrm{f}}$ the rate of change of the fluid domain, the velocity field $\boldsymbol{u}$ and the pressure $p$ satisfy the momentum and continuity equations:

$$
\begin{aligned}
& \rho_{\mathrm{f}}\left(\left.\frac{\partial \boldsymbol{u}}{\partial t}\right|_{x_{0}}+\left(\boldsymbol{u}-\boldsymbol{w}^{\mathrm{f}}\right) \cdot \boldsymbol{\nabla} \boldsymbol{u}\right)-\operatorname{div}\left[\boldsymbol{\sigma}_{\mathrm{f}}(\boldsymbol{u}, p)\right]=\boldsymbol{f}_{\mathrm{f}} \text { in } \Omega^{\mathrm{f}}(t), \\
& \operatorname{div} \boldsymbol{u}=0 \quad \text { in } \Omega^{\mathrm{f}}(t), \\
& \boldsymbol{u}=\boldsymbol{u}_{\text {in }} \text { on } \Gamma^{\text {in }}(t), \quad \boldsymbol{\sigma}_{\mathrm{f}}(\boldsymbol{u}, p) \cdot \boldsymbol{n}_{\mathrm{f}}=\boldsymbol{g}_{\mathrm{f}} \text { on } \Gamma^{\text {out }}(t) .
\end{aligned}
$$

We denote by $\rho_{\mathrm{f}}$ the fluid density, $\mu$ the fluid viscosity, $\boldsymbol{\sigma}_{\mathrm{f}}(\boldsymbol{u}, p)=-p I d+$ $2 \mu \boldsymbol{\epsilon}(\boldsymbol{u})$ the Cauchy stress tensor, Id is the identity matrix, $\boldsymbol{\epsilon}(\boldsymbol{u})=(\boldsymbol{\nabla} \boldsymbol{u}+$ 
$\left.(\boldsymbol{\nabla} \boldsymbol{u})^{T}\right) / 2$ the strain rate tensor. Note that (3) does not define univocally a solution $(\boldsymbol{u}, p)$ as no boundary data are prescribed on the interface $\Gamma(t)$.

Similarly, for given vector functions $\boldsymbol{g}_{\mathrm{s}}, \boldsymbol{f}_{\mathrm{s}}$, we consider the following structure problem whose solution is $\mathrm{d}^{\mathrm{s}}$ :

$$
\begin{aligned}
\rho_{\mathrm{s}} \frac{\partial^{2} \boldsymbol{d}^{\mathrm{s}}}{\partial t^{2}}-\operatorname{div}_{\mid x_{0}}\left(\boldsymbol{\sigma}_{\mathrm{s}}\left(\boldsymbol{d}^{\mathrm{s}}\right)\right) & =\boldsymbol{f}_{\mathrm{s}} \text { in } \Omega_{0}^{\mathrm{s}}, \\
\boldsymbol{\sigma}_{\mathrm{s}}\left(\boldsymbol{d}^{\mathrm{s}}\right) \cdot \boldsymbol{n}_{\mathrm{s}} & =\boldsymbol{g}_{\mathrm{s}} \text { on } \partial \Omega_{0}^{\mathrm{s}} \backslash \Gamma_{0},
\end{aligned}
$$

where $\boldsymbol{\sigma}_{\mathrm{s}}\left(\boldsymbol{d}^{\mathrm{s}}\right)$ is the first Piola-Kirchoff stress tensor. We remark that boundary values on $\Gamma_{0}$ for (4) are missing.

When coupling the two problems together, the "missing" boundary conditions are indeed supplemented by suitable matching conditions on the reference interface $\Gamma_{0}$. If $\lambda=\lambda(t)$ denotes the displacement of the interface, at any time $t$ the coupling conditions on the reference interface $\Gamma_{0}$ are

$$
\begin{gathered}
\boldsymbol{x}_{t}^{\mathrm{s}}=x_{0}+\lambda=\boldsymbol{x}_{t}^{\mathrm{f}}, \quad \boldsymbol{u} \circ \boldsymbol{x}_{t}^{\mathrm{f}}=\frac{\partial \lambda}{\partial t}, \\
\left(\boldsymbol{\sigma}_{\mathrm{f}}(\boldsymbol{u}, p) \cdot \boldsymbol{n}_{\mathrm{f}}\right) \circ \boldsymbol{x}_{t}^{\mathrm{f}}=-\boldsymbol{\sigma}_{\mathrm{s}}\left(\boldsymbol{d}^{\mathrm{s}}\right) \cdot \boldsymbol{n}_{\mathrm{s}},
\end{gathered}
$$

imposing the matching of the interface displacements of the fluid and solid subdomains, the continuity of the velocities and of the normal stresses.

\section{The interface equations associated to problem (3)-(5)}

We consider the coupled problem at a given time $t=t^{n+1}=(n+1) \delta t, \delta t$ being the discrete time-step.

According to the interface conditions (5), we can envisage two possible natural choices for the interface variable: either we consider the displacement $\lambda$ of the fluid-structure interface, or the normal stress exerted on it. In the following, we shall focus our attention on the case of the interface variable as the displacement; the "dual" approach using the normal stress was presented in [4] for a simple linear problem.

Thus, we define the fluid and structure interface operators as follows.

$S_{\mathrm{f}}$ is the Dirichlet-to-Neumann map in $\Omega^{\mathrm{f}}(t)$ :

$$
S_{\mathrm{f}}: H^{1 / 2}\left(\Gamma_{0}\right) \rightarrow H^{-1 / 2}\left(\Gamma_{0}\right), \quad \lambda \rightarrow \sigma_{\mathrm{f}}(\lambda),
$$

that operates between the trace space of displacements on the interface $\Gamma_{0}$ and the dual space of the normal stresses exerted on $\Gamma_{0}$ by the fluid. Computing $S_{\mathrm{f}}(\lambda)$ involves the extension of the interface displacement to the whole fluid domain (in order to compute the ALE velocity), the solution of a NavierStokes problem in $\Omega^{\mathrm{f}}(t)$ with the Dirichlet boundary condition on the interface $\boldsymbol{u}_{\mid \Gamma(t)} \circ \boldsymbol{x}_{t}^{\mathrm{f}}=\left(\lambda-\mathrm{d}_{\mid \Gamma_{0}}^{\mathrm{s}, n}\right) / \delta t$, and then to recover the normal stress $\sigma_{\mathrm{f}}=\left(\boldsymbol{\sigma}_{\mathrm{f}}(\boldsymbol{u}, p)\right.$. $\left.\boldsymbol{n}_{\mathrm{f}}\right)_{\mid \Gamma(t)} \circ \boldsymbol{x}_{t}^{\mathrm{f}}$ as a residual of the Navier-Stokes equations on the interface. 
Moreover, we consider the Dirichlet-to-Neumann map $S_{\mathrm{s}}$ in $\Omega_{0}^{\mathrm{s}}$ :

$$
S_{\mathrm{s}}: H^{1 / 2}\left(\Gamma_{0}\right) \rightarrow H^{-1 / 2}\left(\Gamma_{0}\right), \quad \lambda \rightarrow \sigma_{\mathrm{s}}(\lambda),
$$

that operates between the space of displacements on the interface $\Gamma_{0}$ and the space of the normal stresses exerted by the structure on $\Gamma_{0}$. Computing $S_{\mathrm{s}}(\lambda)$ corresponds to solving a structure problem in $\Omega_{0}^{\mathrm{s}}$ with Dirichlet boundary condition $\mathrm{d}_{\mid \Gamma_{0}}^{\mathrm{s}}=\lambda$ on $\Gamma_{0}$, and then to recover the normal stress $\sigma_{\mathrm{s}}=\boldsymbol{\sigma}_{\mathrm{s}}\left(\mathrm{d}^{\mathrm{s}}\right) \cdot \boldsymbol{n}_{\mathrm{s}}$ on the interface, again as a residual.

The definitions of $S_{\mathrm{f}}$ and $S_{\mathrm{s}}$ involve also the boundary and forcing terms, because of the nonlinearity of the problem at hand.

Then, the coupled fluid-structure problem can be expressed in terms of the solution $\lambda$ of the following nonlinear Steklov-Poincaré interface problem:

$$
\text { find } \lambda \in H^{1 / 2}\left(\Gamma_{0}\right): \quad S_{\mathrm{f}}(\lambda)+S_{\mathrm{s}}(\lambda)=0 .
$$

Remark 1. In the case of a linear coupled Stokes-shell model, Mouro [19] has given a precise characterization of these interface operators and shown that they are selfadjoint and positive.

The inverse operator $S_{\mathrm{s}}^{-1}$ is a Neumann-to-Dirichlet map that for any given normal stress $\sigma$ on $\Gamma_{0}$ associates the interface displacement $\lambda\left(t^{n+1}\right)=\mathrm{d}^{\mathrm{s}, n+1}$ by solving a structure problem with the Neumann boundary condition $\sigma_{\mathrm{s}}\left(\mathrm{d}^{s}\right)$. $\boldsymbol{n}_{\mathrm{s}}=\sigma$ on $\Gamma_{0}$ and then computing the restriction on $\Gamma_{0}$ of the displacement of the structure domain.

For nonlinear structural models (i.e. $\sigma_{\mathrm{s}}\left(\mathrm{d}^{s}\right)$ is a nonlinear constitutive law in (4), see, e.g., [17]), we will need the tangent operator $S_{\mathrm{s}}^{\prime}$

$$
S_{\mathrm{s}}^{\prime}(\bar{\lambda}) \delta \lambda=\lim _{h \rightarrow 0} \frac{S_{\mathrm{s}}(\bar{\lambda}+h \delta \lambda)-S_{\mathrm{s}}(\bar{\lambda})}{h}, \quad \forall \bar{\lambda}, \delta \lambda \in H^{1 / 2}\left(\Gamma_{0}\right) .
$$

Its inverse $\left(S_{\mathrm{s}}^{\prime}\right)^{-1}$ is a Neumann-to-Dirichlet map that for any given variation of the normal stress $\delta \sigma$ on $\Gamma_{0}$ associates the corresponding variation of the displacement $\delta \lambda$ of the interface by solving a linearized structure problem with boundary condition $\boldsymbol{\sigma}_{\mathrm{s}}\left(\boldsymbol{d}^{\mathrm{s}}\right) \cdot \boldsymbol{n}_{\mathrm{s}}=\delta \sigma$ on $\Gamma_{0}$. Similarly, we define $S_{\mathrm{f}}^{\prime}$ by

$$
S_{\mathrm{f}}^{\prime}(\bar{\lambda}) \delta \lambda=\lim _{h \rightarrow 0} \frac{S_{\mathrm{f}}(\bar{\lambda}+h \delta \lambda)-S_{\mathrm{f}}(\bar{\lambda})}{h}, \quad \forall \bar{\lambda}, \delta \lambda \in H^{1 / 2}\left(\Gamma_{0}\right) .
$$

This is a Dirichlet-to-Neumann map that for any variation of the interface displacement $\delta \lambda$ computes the corresponding variation of the normal stress $\delta \sigma$ on $\Gamma_{0}$ through the solution of linearized Navier-Stokes equations. To compute $S_{\mathrm{f}}^{\prime}(\lambda) \delta \lambda$ see, e.g, $[9]$.

The computation of the inverse operator $S_{\mathrm{f}}^{\prime}(\lambda)^{-1}$ can be simplified by neglecting the shape derivatives. We then obtain the Oseen equations in the fixed configuration defined by $\lambda$ that we computed while evaluating $S_{\mathrm{f}}(\lambda)$. $S_{\mathrm{f}}^{\prime}(\lambda)^{-1}$ is a Neumann-to-Dirichlet map that for any given variation of the 
normal stress $\delta \sigma$ on $\Gamma_{0}$ computes the corresponding displacement $\delta \lambda$ of the interface through the solution of linearized Navier-Stokes equations with the boundary condition $\left(\boldsymbol{\sigma}_{\mathrm{f}}(\boldsymbol{u}, p) \cdot \boldsymbol{n}_{\mathrm{f}}\right) \circ \boldsymbol{x}^{\mathrm{f}}=\sigma$ on $\Gamma_{0}$.

Other possible formulations for the interface equation can be given:

$$
\text { find } \lambda \text { such that } S_{\mathrm{s}}^{-1}\left(-S_{\mathrm{f}}(\lambda)\right)=\lambda \text { on } \Gamma_{0} \text {, }
$$

or equivalently

$$
\text { find } \lambda \text { such that } S_{\mathrm{s}}^{-1}\left(-S_{\mathrm{f}}(\lambda)\right)-\lambda=0 \text { on } \Gamma_{0} \text {. }
$$

These are common formulations in fluid-structure interaction problems, but it is worth pointing out that here the unknown $\lambda$ is the displacement of the sole interface, whereas classically the displacement of the whole solid domain is considered (see, e.g., $[20,9]$ ).

\section{Iterative methods for problems (6)-(8)}

We consider the preconditioned Richardson method to solve the SteklovPoincaré interface problem (6): given $\lambda^{0}$, for $k \geq 0$, solve

$$
P_{k}\left(\lambda^{k+1}-\lambda^{k}\right)=\omega^{k}\left(-S_{\mathrm{f}}\left(\lambda^{k}\right)-S_{\mathrm{s}}\left(\lambda^{k}\right)\right) .
$$

The scaling operator $P_{k}$ maps the space $H^{1 / 2}\left(\Gamma_{0}\right)$ of the interface variable onto the space $H^{-1 / 2}\left(\Gamma_{0}\right)$ of normal stresses, and may depend on the iterate $\lambda^{k}$ or, more generally, on the iteration step $k$. The acceleration parameter $\omega^{k}$ can be computed via the Aitken technique (see [4]) or by line search (see [22]).

At each step $k,(9)$ requires the solution, separately, of the fluid and the structure problems and then to apply a scaling operator. Precisely,

1. apply $S_{\mathrm{f}}$ to $\lambda^{k}$, i.e., compute the extension of $\lambda^{k}$ to the entire fluid domain to obtain the ALE velocity, and solve the fluid problem in $\Omega^{\mathrm{f}}(t)$ with boundary condition $\boldsymbol{u}_{\mid \Gamma(t)} \circ \boldsymbol{x}_{t}^{\mathrm{f}}=\left(\lambda-\mathrm{d}_{\mid \Gamma_{0}}^{\mathrm{s}, n}\right) / \delta t$ on $\Gamma_{0}$; then, recover the normal stress $\sigma_{\mathrm{f}}^{k}$ on the interface;

2. apply $S_{\mathrm{s}}$ to $\lambda^{k}$, i.e., solve the structure problem with boundary condition $\mathrm{d}_{\mid \Gamma(t)}^{\mathrm{s}, k}=\lambda^{k}$ on $\Gamma(t)$ and compute the normal stress $\sigma_{\mathrm{s}}^{k}$;

3. apply $P_{k}^{-1}$ to the total stress $\sigma^{k}=\sigma_{\mathrm{f}}^{k}+\sigma_{\mathrm{s}}^{k}$ on the interface.

Note that steps 1 and 2 can be performed in parallel. The crucial issue is how to choose the scaling operator (more precisely, a preconditioner in the finite dimensional case) in order for the iterative method to converge as quickly as possible.

We define a generic linear operator (more precisely, its inverse):

$$
P_{k}^{-1}=\alpha_{\mathrm{f}}^{k} S_{\mathrm{f}}^{\prime}\left(\lambda^{k}\right)^{-1}+\alpha_{\mathrm{s}}^{k} S_{\mathrm{s}}^{\prime}\left(\lambda^{k}\right)^{-1},
$$


for two given scalars $\alpha_{\mathrm{f}}^{k}$ and $\alpha_{\mathrm{s}}^{k}$, and we retrieve the following operators:

Dirichlet-Neumann (DN): $P_{k}=P_{D N}=S_{\mathrm{s}}^{\prime}\left(\lambda^{k}\right)$, for $\alpha_{\mathrm{f}}^{k}=0, \alpha_{\mathrm{s}}^{k}=1$,

Neumann-Dirichlet $(N D): P_{k}=P_{N D}=S_{\mathrm{f}}^{\prime}\left(\lambda^{k}\right)$, for $\alpha_{\mathrm{f}}^{k}=1, \alpha_{\mathrm{s}}^{k}=0$,

Neumann-Neumann (NN): $P_{k}=P_{N N}$ with $\alpha_{\mathrm{f}}^{k}+\alpha_{\mathrm{s}}^{k}=1, \alpha_{\mathrm{f}}^{k}, \alpha_{\mathrm{s}}^{k} \neq 0$.

If the structure is linear, the computational effort of a Richardson step in the DN case may be reduced to the solution of only one fluid Dirichlet problem and one structure Neumann problem.

The parameters $\alpha_{\mathrm{f}}^{k}, \alpha_{\mathrm{s}}^{k}$ and $\omega^{k}$ can be chosen dynamically using a generalized Aitken technique (see [3, 4]).

Should we consider the scaling operator

$$
P_{k}=S_{\mathrm{f}}^{\prime}\left(\lambda^{k}\right)+S_{\mathrm{s}}^{\prime}\left(\lambda^{k}\right),
$$

then, we would retrieve the genuine Newton algorithm applied to the SteklovPoincaré problem (6). Note that in order to perform the scaling step 3 in the Richardson algorithm, one must use a (preconditioned) iterative method (e.g., GMRES) and may approximate the tangent problems to accelerate the computations. Thus, using the scaling operator (14) we obtain a domain decomposition-Newton (DD-Newton) method; more precisely, given a solid state displacement $\lambda^{k}$, for $k \geq 0$, the algorithm reads

1. solve the fluid and the structure subproblems separately, as for the Richardson method, to get $\sigma^{k}$;

2. solve the following linear system via GMRES to compute $\mu^{k}$ :

$$
\left[S_{\mathrm{f}}^{\prime}\left(\lambda^{k}\right)+S_{\mathrm{s}}^{\prime}\left(\lambda^{k}\right)\right] \mu^{k}=-\left(S_{\mathrm{f}}\left(\lambda^{k}\right)+S_{\mathrm{s}}\left(\lambda^{k}\right)\right)
$$

3. update the displacement: $\lambda^{k+1}=\lambda^{k}+\omega^{k} \mu^{k}$.

The GMRES solver should in turn be preconditioned in order to accelerate its convergence rate. To this aim, one can use one of the previously defined scaling operators. In our numerical tests, we have considered the DN operator $S_{\mathrm{s}}^{\prime}(\lambda)$, so that the preconditioned matrix of the GMRES method becomes:

$$
\left[S_{\mathrm{s}}^{\prime}\left(\lambda^{k}\right)\right]^{-1} \cdot\left[S_{\mathrm{f}}^{\prime}\left(\lambda_{k}\right)+S_{\mathrm{s}}^{\prime}\left(\lambda_{k}\right)\right] .
$$

Let us briefly recall the Newton method for problem (8) in order to compare it with the previous domain decomposition approach. For a more complete discussion we refer to [4].

Let $J(\lambda)$ denote the Jacobian of $S_{\mathrm{s}}^{-1}\left(-S_{\mathrm{f}}(\lambda)\right)$ in $\lambda$. Given $\lambda^{0}$, for $k \geq 0$ :

$$
\begin{aligned}
& \text { solve }\left(J\left(\lambda^{k}\right)-I d\right) \mu^{k}=-\left(S_{\mathrm{s}}^{-1}\left(-S_{\mathrm{f}}\left(\lambda^{k}\right)\right)-\lambda^{k}\right), \\
& \text { update } \quad \lambda^{k+1}=\lambda^{k}+\omega^{k} \mu^{k} .
\end{aligned}
$$

The parameter $\omega^{k}$ can be computed, e.g., by a line search technique (see [22]). Note that the Jacobian in $\lambda^{k}$ has the following expression: 


$$
J\left(\lambda^{k}\right)=-\left[S_{\mathrm{s}}^{\prime}\left(S_{\mathrm{s}}^{-1}\left(-S_{\mathrm{f}}\left(\lambda^{k}\right)\right)\right)\right]^{-1} \cdot S_{\mathrm{f}}^{\prime}\left(\lambda^{k}\right)=-\left[S_{\mathrm{s}}^{\prime}\left(\bar{\lambda}^{k}\right)\right]^{-1} \cdot S_{\mathrm{f}}^{\prime}\left(\lambda^{k}\right) .
$$

The solution of the linear system (17) can be obtained by using an iterative matrix-free method such as GMRES.

In general, the Newton method applied to (8) and to the Steklov-Poincaré formulation (6) are not equivalent. However, in the case of a linear structure, they actually are (to see this, left multiply both hand sides of (15) by $S_{\mathrm{s}}^{-1}$, exploit $S_{\mathrm{s}}^{\prime}\left(\lambda^{k}\right)=S_{\mathrm{s}}$ and compare (16) with (17)).

We remark that while the computation of $\left[S_{\mathrm{s}}^{\prime}\left(\bar{\lambda}^{k}\right)\right]^{-1} \cdot \delta \sigma$ (for any given $\delta \sigma$ ) does only require the derivative with respect to the state variable at the interface, the computation of $S_{\mathrm{f}}^{\prime}\left(\lambda^{k}\right) \cdot \delta \lambda$ is nontrivial since it also requires shape derivatives, as a variation in $\lambda$ determines a variation of the fluid domain.

We finally remark that in the classical Newton method, the fluid and structure problems must be solved separately and sequentially, while the domain decomposition formulation allows us to set up parallel algorithms to solve the Steklov-Poincaré equation (6).

\section{Numerical results}

In this section, we present some numerical results which compare the domain decomposition methods to the classical fixed point and Newton algorithms, and illustrate their behavior with respect to the grid size $h$ and the time step $\delta t$.

For the domain decomposition algorithms, we consider the DN preconditioner (11), and the NN preconditioner (13) in which $S_{\mathrm{f}}^{\prime}$ is linearized by neglecting the shape derivatives.

Finally, we consider the DD-Newton method (14). The fluid tangent problem is considered as in [9] in its exact form. To solve (15), we apply the GMRES method possibly preconditioned by the operator DN (11).

Both problems (3) and (4) are discretized, and we adopt $\mathbb{P}_{1}$-bubble $/ \mathbb{P}_{1}$ finite elements for the fluid and $\mathbb{P}_{1}$ elements for the structure. The simulations are performed on a dual $2.8 \mathrm{Ghz}$ Pentium 4 Xeon with 3 GB of RAM.

We simulate a pressure wave in a straight cylinder of length $5 \mathrm{~cm}$ and radius $5 \mathrm{~mm}$ at rest. The structure of thickness $0.5 \mathrm{~mm}$ is linear and clamped at both the inlet and the outlet. The fluid viscosity is set to $\mu=0.03$ poise, the densities to $\rho_{\mathrm{f}}=1 \mathrm{~g} / \mathrm{cm}^{3}$ and $\rho_{\mathrm{s}}=1.2 \mathrm{~g} / \mathrm{cm}^{3}$. We impose zero body forces and homogeneous Dirichlet boundary conditions on $\partial \Omega_{0}^{\mathrm{s}} \backslash \Gamma_{0}$. The fluid and the structure are initially at rest and a pressure (a normal stress, actually) of $1.3332 \cdot 10^{4}$ dynes $/ \mathrm{cm}^{2}$ is imposed on the inlet for $3 \cdot 10^{-3} \mathrm{~s}$. We consider two computational meshes: a coarse one with 1050 nodes (4680 elements) for the fluid and 1260 nodes (4800 elements) for the solid, and a finer mesh with 2860 nodes (14100 elements) for the fluid and 2340 nodes (9000 elements) for the solid. 
A comparison between the fixed point iterations for problem (7) and Richardson iterations (9) (with DN and NN preconditioners) on problem (6) is shown in table 1 for two time steps and for the coarse and the fine mesh. In this table, "FS eval" stands for the average number of evaluations per time step of either (7) or (9), while "FS' eval" represents the average number of evaluations of the corresponding linearized system per time step (that is (10) for DN, ND or NN preconditioners, (16) for the DD-Newton method (15), and (18) for the classical Newton method (17)). We can see that, using the preconditioned Richardson method (9), fewer FS evaluations than with the classical fixed point algorithm are needed. However, the computational time of the domain decomposition formulation is slightly higher than that of the fixed point formulation. The reason is that the domain decomposition formulation requires solving, at each iteration, the fluid and the structure subproblems, as well as the associated tangent problems, while the latter are indeed skipped by the fixed point procedure. Furthermore, since the operator for the structure is linear, the two approaches are very similar and since our research code is sequential, the parallel structure of the Steklov-Poincaré formulation (6) is not capitalized.

Moreover, we notice that using the NN preconditioner the number of iterations required for the convergence with respect to both parameters $h$ and $\delta t$, does not vary appreciably.

The same table shows also the results obtained using the Newton and DDNewton methods. The Jacobian matrices (14) and (18) have been computed exactly (see [9]) and inverted by GMRES. The number of iterations of Newton and DD-Newton is equivalent, but the inversion of the Jacobian in DD-Newton ("FS' eval") needs more GMRES iterations, a number which depends on $h$ and $\delta t$. However, preconditioning GMRES by DN reduces the iteration numbers to the same as in Newton, and the CPU times are then quite similar. As before, the reasons reside in the linearity of the structure model and in the fact that our code is sequential.

Further improvements may be obtained resorting to more sophisticated preconditioners for the Jacobian system, derived either from the classical domain decomposition theory or from lower dimensional models (in a multiscale approach, see [21]).

We now simulate a pressure wave in the carotid bifurcation using the same fluid and structure characteristics as before. We solve the coupling using our DD-Newton algorithm with DN preconditioner for the GMRES inner iterations. The mesh that we have used was computed using an original realistic geometry first proposed in [15].

The fluid and the structure are initially at rest and a pressure of 1.3332 . $10^{4}$ dynes $/ \mathrm{cm}^{2}$ is set at the inlet for a time of $3 \cdot 10^{-3} \mathrm{~s}$. The average inflow diameter is $0.67 \mathrm{~cm}$, the time step used is $\delta t=1 e-04$ and the total number of iterations is 200. Figure 2 displays the solution computed at two different time steps. Table 2 shows the comparison between the classical Newton algorithm and our DD-Newton algorithm preconditioned by DN. Like in 
Table 1. Comparison of the number of sub-iterations and computational time for the fixed point, and domain decomposition based algorithms for the coarse mesh (left) and fine mesh (right)

\begin{tabular}{|c|c|c|c|}
\hline \multicolumn{4}{|c|}{$\delta t=0.001$} \\
\hline Method & FS eval & FS' eval & CPU time \\
\hline Fixed point & $\mid \overline{|c|}$ & 0 & $\overline{1 \mathrm{~h} 166^{\prime}}$ \\
\hline $\mathrm{DN}$ & 19.8 & 19.8 & 1h17' \\
\hline NN & 17.9 & 17.9 & $1 \mathrm{~h} 42^{\prime}$ \\
\hline Newton & 3 & 12 & 0h56' \\
\hline DD-Newton & 3 & 24 & $1 \mathrm{~h} 30^{\prime}$ \\
\hline DD-Newton DN & 3 & 12 & $0 \mathrm{~h} 58^{\prime}$ \\
\hline \multicolumn{4}{|c|}{$\delta t=0.0005$} \\
\hline Method & \multicolumn{3}{|c|}{ |FS eval|FS' eval|CPU time } \\
\hline Fixed point & 32.1 & 0 & $3 \mathrm{~h}^{2} 27^{\prime}$ \\
\hline $\mathrm{DN}$ & 29.2 & 29.2 & $3 \mathrm{~h} 50$ ' \\
\hline NN & 22 & 22 & $4 \mathrm{~h} 20$ ' \\
\hline Newton & 3 & 17 & $1 \mathrm{~h} 55^{\prime}$ \\
\hline DD-Newton & 3 & 29 & $3 \mathrm{~h} 30^{\prime}$ \\
\hline \begin{tabular}{|l|} 
DD-Newton DN \\
\end{tabular} & 3 & 17 & $2 \mathrm{~h} 10^{\prime}$ \\
\hline \multicolumn{4}{|c|}{$\delta t=0.0001$} \\
\hline Method & FS eval & FS' eval & CPU time \\
\hline Newton & 3 & 19 & $11 \mathrm{~h} 411$ \\
\hline DD-Newton & 3 & 35 & $16 \mathrm{~h}^{2} 1^{\prime}$ \\
\hline \begin{tabular}{|l|} 
DD-Newton DN \\
\end{tabular} & 3 & 19 & $12 \mathrm{~h} 39^{\prime}$ \\
\hline
\end{tabular}

\begin{tabular}{|l|r|r|r|}
\hline \multicolumn{4}{|c|}{$\delta t=0.001$} \\
\hline \hline Method & FS eval & FS' eval & CPU time \\
\hline \hline Fixed point & 19.9 & 0 & $4 \mathrm{~h} 28^{\prime}$ \\
\hline DN & 19.5 & 19.5 & $4 \mathrm{~h} 40^{\prime}$ \\
\hline NN & 17.7 & 17.7 & $6 \mathrm{~h}^{\prime}$ \\
\hline Newton & 3 & 12 & $3 \mathrm{~h} 39^{\prime}$ \\
\hline DD-Newton & 3 & 30 & $4 \mathrm{~h} 56^{\prime}$ \\
\hline DD-Newton DN & 3 & 12 & $3 \mathrm{~h} 45^{\prime}$ \\
\hline \hline \multicolumn{3}{|c|}{$\delta t=0.0005$} \\
\hline \hline Method & FS eval & FS' eval & CPU time \\
\hline \hline Fixed point & 33 & 0 & $12 \mathrm{~h} 40^{\prime}$ \\
\hline DN & 29.6 & 29.6 & $12 \mathrm{~h} 50^{\prime}$ \\
\hline NN & 22.1 & 22.1 & $15 \mathrm{~h} 44^{\prime}$ \\
\hline Newton & 3 & 14 & $8 \mathrm{~h} 31^{\prime}$ \\
\hline DD-Newton & 3 & 35 & $10 \mathrm{~h} 50^{\prime}$ \\
\hline DD-Newton DN & 3 & 14 & $8 \mathrm{~h} 40^{\prime}$ \\
\hline \hline \multicolumn{4}{|c|}{$\delta t=0.0001$} \\
\hline \hline Method & FS eval & FS' eval & CPU time \\
\hline \hline Newton & 3 & 19 & $26 \mathrm{~h} 40 \prime$ \\
\hline DD-Newton & 3 & 37 & $40 \mathrm{~h} 26^{\prime}$ \\
\hline DD-Newton DN & 3 & 19 & $27 \mathrm{~h} 01^{\prime}$ \\
\hline
\end{tabular}

the previous test, "FS eval" and "FS' eval" represent respectively the average number of fluid/structure evaluations and the average number of linearized fluid/structure evaluations. As expected, both methods behave in the same way with respect to the number of operator evaluations. The total computation times are also in very good agreement for the two largest time step.
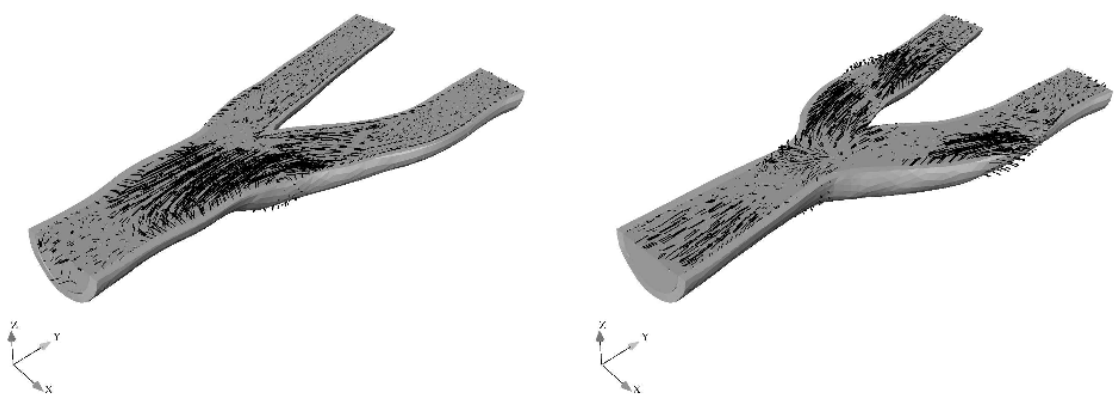

Fig. 2. Structure deformation and fluid velocity at $t=0.005 \mathrm{~s}$ (left) and $t=0.008 \mathrm{~s}$ (right) 
Table 2. Convergence comparison of the computational time for the exact Newton and DD-Newton methods (case of carotid bifurcation)

\begin{tabular}{|c|c|c|c|c|c|c|c|c|c|}
\hline & \multicolumn{3}{|c|}{$\delta t=0.001$} & \multicolumn{3}{|c|}{$\delta t=0.0005$} & \multicolumn{3}{|c|}{$\delta t=0.0001$} \\
\hline Method & FS eval & FS' eval & CPU time & FS eval & FS' eval & CPU time & FS eval & \begin{tabular}{|l|} 
FS' eval \\
\end{tabular} & CPU time \\
\hline Newton & 3 & 7.5 & $8 \mathrm{~h} 51$ ' & 3 & 10 & 19h41' & 3 & 19 & $125 \mathrm{~h} 20^{\prime}$ \\
\hline DD-Newton DN & 3 & 7.5 & $8 \mathrm{~h} 12$ & 3 & 10 & 19h33' & 3 & 19 & 131h08' \\
\hline
\end{tabular}

Acknowledgement. This research has been supported by the Swiss National Science Foundation (project 20-101-800) and by the INDAM project "Integrazione di sistemi complessi in biomedicina: modelli, simulazioni, rappresentazioni".

\section{References}

1. P. Causin, J.-F. Gerbeau, and F. Nobile, Added-mass effect in the design of partitioned algorithms for fluid-structure problems, Comput. Methods Appl. Mech. Engrg., 194 (2005), pp. 4506-4527.

2. M. Cervera, R. Codina, And M. Galindo, On the computational efficiency and implementation of block-iterative algorithms for nonlinear coupled problems, Engrg. Comput., 13 (1996), pp. 4-30.

3. S. Deparis, Numerical Analysis of Axisymmetric Flows and Methods for FluidStructure Interaction Arising in Blood Flow Simulation, PhD thesis, École Polytechnique Fédérale de Lausanne, 2004.

4. S. Deparis, M. Discacciati, and A. Quarteroni, A domain decomposition framework for fluid-structure interaction problems, in Proceedings of the Third International Conference on Computational Fluid Dynamics, C. Groth and D. W. Zingg, eds., Springer, May 2006.

5. M. DiscacCIATI, Domain Decomposition Methods for the Coupling of Surface and Groundwater Flows, $\mathrm{PhD}$ thesis, École Polytechnique Fédérale de Lausanne, 2004.

6. J. DoneA, Arbitrary Lagrangian Eulerian finite element methods, in Computational Methods for Transient Analysis, vol. 1 of Computational Methods in Mechanics, Amsterdam, North-Holland, 1983, pp. 473-516.

7. L. Fatone, P. Gervasio, And A. QuARTERoni, Multimodels for incompressible flows, J. Math. Fluid Mech., 2 (2000), pp. 126-150.

8. - Multimodels for incompressible flows: iterative solutions for the NavierStokes/Oseen coupling, Math. Model. Numer. Anal., 35 (2001), pp. 549-574.

9. M. A. Fernández AND M. Moubachir, A Newton method using exact Jacobians for solving fluid-structure coupling, Comput. \& Structures, 83 (2005), pp. 127-142.

10. J. C. Galvis and M. Sarkis, Inf-sup for coupling Stokes-Darcy, in Proceedings of the XXV Iberian Latin American Congress in Computational Methods in Engineering, A. L. et al., ed., Universidade Federal de Pernambuco, 2004.

11. F. Gastaldi, A. Quarteroni, And G. S. Landriani, On the coupling of two-dimensional hyperbolic and elliptic equations: analytical and numerical approach, in Third International Symposium on Domain Decomposition Methods 
for Partial Differential Equations, held in Houston, Texas, March 20-22, 1989, T. F. Chan, R. Glowinski, J. Périaux, and O. Widlund, eds., Philadelphia, PA, 1990, SIAM, pp. 22-63.

12. J.-F. Gerbeau And M. Vidrascu, A quasi-Newton algorithm based on a reduced model for fluid-structure interaction problems in blood flows, Math. Model. Numer. Anal., 37 (2003), pp. 631-647.

13. M. HEIL, An efficient solver for the fully coupled solution of large-displacement fluid-structure interaction problems, Comput. Methods Appl. Mech. Engrg., 193 (2004), pp. 1-23.

14. T. J. Hughes, W. K. Liu, And T. K. Zimmermann, Lagrangian-Eulerian finite element formulation for incompressible flows, Comput. Methods Appl. Mech. Engrg., 29 (1981), pp. 329-349.

15. G. Karner, K. Perktold, M. Hofer, And D. Liepsch, Flow characteristics in an anatomically realistic compliant carotid artery bifurcation model, Comput. Methods Biomech. Biomed. Engrg., 2 (1999), pp. 171-185.

16. W. J. Layton, F. Schieweck, And I. Yotov, Coupling fluid flow with porous media flow, SIAM J. Num. Anal., 40 (2003), pp. 2195-2218.

17. J. E. Marsden And T. J. Hughes, Mathematical Foundations of Elasticity, Dover Publications, Inc., New York, 1994. Reprint.

18. D. P. MOK AND W. A. WALL, Partitioned analysis schemes for the transient interaction of incompressible flows and nonlinear flexible structures, in Proceedings of the International Conference Trends in Computational Structural Mechanics, K. Schweizerhof and W. A. Wall, eds., K.U. Bletzinger, CIMNE, Barcelona, 2001.

19. J. Mouro, Interactions Fluide Structure en Grands Déplacements. Résolution Numérique et Application aux Composants Hydrauliques Automobiles, $\mathrm{PhD}$ thesis, École Polytechnique, Paris, September 1996.

20. F. NobiLe, Numerical Approximation of Fluid-Structure Interaction Problems with Application to Haemodynamics, $\mathrm{PhD}$ thesis, École Polytechnique Fédérale de Lausanne, 2001.

21. A. QuARteroni And L. Formaggia, Mathematical modelling and numerical simulation of the cardiovascular system, in Modelling of Living Systems, P. G. Ciarlet and J. L. Lions, eds., vol. 12 of Handbook of Numerical Analysis, Elsevier, Amsterdam, 2004, pp. 3-127.

22. A. Quarteroni, R. Sacco, And F. Saleri, Numerical Mathematics, Texts in Applied Mathematics, Springer, New York, 2000.

23. A. Quarteroni, A. Veneziani, And P. Zunino, A domain decomposition method for advection-diffusion processes with application to blood solutes, SIAM J. Sci. Comput., 23 (2002), pp. 1959-1980.

24. P. L. TAlleC AND J. Mouro, Fluid structure interaction with large structural displacements, Comput. Methods Appl. Mech. Engrg., 190 (2001), pp. 30393067.

25. P. Zunino, Iterative substructuring methods for advection-diffusion problems in heterogeneous media, in Challenges in Scientific Computing-CISC 2002, vol. 35 of Lecture Notes in Computational Science and Engineering, Springer, 2003, pp. $184-210$. 